\title{
Muséologies
}

Les cahiers d'études supérieures

muséologies

\section{Laurier Lacroix}

\section{Alessandra Mariani}

Volume 8, numéro 2, 2016

URI : https://id.erudit.org/iderudit/1050768ar

DOI : https://doi.org/10.7202/1050768ar

Aller au sommaire du numéro

Éditeur(s)

Association Québécoise de Promotion des Recherches Étudiantes en Muséologie (AQPREM)

ISSN

1718-5181 (imprimé)

1929-7815 (numérique)

Découvrir la revue

Citer ce document

Mariani, A. (2016). Laurier Lacroix. Muséologies, 8(2), 193-210.

https://doi.org/10.7202/1050768ar

Tous droits réservés (C Association Québécoise de Promotion des Recherches Étudiantes en Muséologie (AQPREM), 2018
Ce document est protégé par la loi sur le droit d'auteur. L’utilisation des services d'Érudit (y compris la reproduction) est assujettie à sa politique d'utilisation que vous pouvez consulter en ligne.

https://apropos.erudit.org/fr/usagers/politique-dutilisation/
Cet article est diffusé et préservé par Érudit.

Érudit est un consortium interuniversitaire sans but lucratif composé de l’Université de Montréal, l'Université Laval et l'Université du Québec à Montréal. Il a pour mission la promotion et la valorisation de la recherche. https://www.erudit.org/fr/ 
Entrevue

\section{Laurier Lacroix}

Alessandra Mariani 
Laurier Lacroix, C.M., est professeur émérite de l'Université du Québec à Montréal où il a enseigné l'histoire de l'art et la muséologie. Détenteur d'une maîtrise en littérature et civilisation françaises (McGill), d'une maîtrise en histoire de l'art (Université de Montréal) et d'un doctorat en histoire (Laval), il a débuté sa carrière d'enseignement à l'Université Concordia. Ses intérêts de recherche portent principalement sur les collections publiques et l'art au Québec et au Canada avant 1940 et l'historiographie de l'histoire de l'art. Parmi ses réalisations, notons les expositions et les catalogues François Baillairgé (1985), Peindre à Montréal entre 1915 et 1930 (1996), les rétrospectives consacrées à Ozias Leduc $(1978,1996)$ et Suzor-Coté $(1986,2002)$, ainsi que Les arts en Nouvelle-France (2012). Il s'intéresse également à l'art contemporain et a agi, entre autres, comme commissaire d'expositions des œuvres d'Irene F. Whittome (1990, 1998, 2004), Pierre Dorion (2002), Guy Pellerin (2004), Robert Wolfe (2006), Micheline Beauchemin (2009), Lisette Lemieux (2016). Récipiendaire du Prix Carrière de la Société des musées québécois (1997) et du Prix Gérard-Morisset (2008), Laurier Lacroix est membre de la Société des Dix (2005) et de l'Académie des lettres du Québec (2012). Il est membre du Conseil du patrimoine culturel du Québec. 
Laurier Lacroix, comment avez-vous tracé votre parcours universitaire et comment celui-ci vous a-t-il mené à la muséologie? La chance a accompagné mon parcours. J'ai grandi dans le milieu rural québécois de l'Après-Guerre, à l'aube de la Révolution tranquille, sans présence de culture savante et où le terme " musée " était inexistant. J'ai par contre grandi en contact avec la nature, entouré d'excellents éducateurs. Le slogan " qui s'instruit, s'enrichit » a trouvé écho chez mes parents qui l'ont interprété dans le sens de l'enrichissement matériel. Étant né dans une famille peu fortunée, je suis d'abord passé par l'École normale, car la pédagogie était une formation professionnelle gratuite à l'époque. Je n'avais pas d'idée précise quant à l'activité qui occuperait ma vie d'adulte et je me suis laissé guider par ma curiosité. J'avais 20 ans lorsque j'ai complété cette première formation et je me suis inscrit à la maîtrise en psychologie à l'Université Laval. Là, les textes de Sigmund Freud m'ont incité à apprendre l'allemand.

\section{Il s'agit donc d'un parcours marqué par des sauts; des sauts intellectuels, disciplinaires et conceptuels...}

La formation en psychologie qu'on offrait à Laval à cette époque était orientée vers la psychologie expérimentale, ce qui ne me convenait pas. C'est lors d'un séjour estival en Allemagne, en 1968, où j'étais allé consolider mes connaissances de la langue que j'ai découvert l'univers des musées et les œuvres d'art. Ce coup de foudre m'a fait amorcer des études collégiales en histoire de l'art dès mon retour au Québec, études que j'ai poursuivies au baccalauréat à Sir George Williams (Concordia) et à la maîtrise à l'Université de Montréal. J'ai amorcé mon doctorat à Paris en 1973, à l'époque des grands rapprochements entre le Québec et la France. J'y ai obtenu un diplôme en muséologie à l'École du Louvre et une formation universitaire à Paris IV où le grand muséologue George Henri Rivière, fondateur du défunt Musée des arts et traditions populaires, enseignait une muséologie de terrain. Il nous a emmenés visiter les premiers écomusées du Creusot et de Rennes, visiter le chantier de Beaubourg et rencontrer ses concepteurs, etc. À l'École du Louvre, ce sont les divers responsables des départements qui exposaient la nature des services liés aux collections de l'institution. Les deux formations étaient complémentaires. Celle du Louvre était orientée vers les sujets institutionnels de base (recherche, conservation, restauration, gestion), tandis que celle de Rivière abordait directement la question des publics, ou le musée comme lieu communautaire.

J'ai beaucoup profité des séminaires de deux conservateurs: Roseline Bacou au Cabinet des dessins du Louvre et Michel Melot au Cabinet des estampes de la Bibliothèque Nationale. Ces rencontres, où nous étions quelques-uns à discuter autour des réalisations artistiques tirées des réserves ont été de formidables leçons sur les manières de regarder et les approches propres à lire les œuvres. Elles sont sans doute pour beaucoup dans mon besoin de contact direct avec celles-ci. 


\section{Avant d'intégrer le corps professoral de l'UQAM, vous avez occupé le poste de conservateur au Musée des beaux-arts de Montréal. Parlez-nous de cette expérience.}

À mon retour à Montréal à l'automne 1976, j'ai obtenu un poste à l'Université Concordia pour enseigner ma spécialité en histoire de l'art, l'art canadien et québécois. Le professeur John Russell Harper avait mis sur pied le premier programme d'histoire de l'art canadien quelques années auparavant, et j'y ai immédiatement été très impliqué. Mon intérêt pour la muséologie a fait en sorte que j'ai organisé certains de mes séminaires afin qu'ils débouchent sur des expositions préparées avec les étudiants. Deux expositions en sont issues. La première a été une exposition itinérante pancanadienne, qui a bénéficé d'une subvention du ministère du Patrimoine canadien. Elle portait sur les dessins d'Ozias Leduc. La seconde exposait les carnets de dessins du sculpteur québécois François Baillargé, qui avait étudié à Paris à la fin du $18^{\mathrm{e}}$ siècle. Dès lors, j'ai cherché à intéresser et à sensibiliser mes étudiants d'histoire de l'art à la muséologie.

\section{De quelle façon?}

En conceptualisant la recherche en histoire de l'art dans une exposition, notamment par l'élaboration d'une thématique, la muséographie, la rédaction des notices et des outils didactiques, de même que la constitution d'un catalogue d'exposition. Puisqu'il y avait encore peu de spécialistes de l'art québécois à la fin des années 1970 et au début des années 1980, nous avons concocté, les étudiants et moi, ces dispositifs ensemble.

En 1978, à la suite d'un départ à la retraite, le directeur du Musée des beaux-arts de Montréal, Jean Trudel, qui connaissait mon champ de recherche, m'a demandé d'occuper le poste de conservateur de l'art canadien. Parce que j'étais déjà engagé dans l'enseignement depuis deux ans, je lui ai proposé d'effectuer cette tâche avec les étudiants. Cela rejoignait ma conception de l'enseignement qui a toujours visé la fréquentation des œuvres et des sources primaires, les archives dont je partage le "goût " avec Arlette Farge.

Avec trois étudiantes par session, nous nous sommes chargés de tout: du réaccrochage de la collection, de l'organisation d'expositions temporaires, de la création d'expositions monographiques sur la collection, de la gestion de deux grandes expositions venues de l'extérieur. La première, créée à la Galerie nationale du Canada portait sur le peintre Joseph Légaré; la seconde, en provenance du Musée des beaux-arts de l'Ontario, présentait la peinture de paysage montréalaise et torontoise, produite entre 1860 et 1880 . Nous traitions tout ce qui rejoignait mon champ d'expertise, incluant les dossiers d'acquisition. Je crois que cela a été très formateur pour ces étudiantes, certaines ayant poursuivi leur carrière en muséologie ou en enseignement de l'histoire de l'art. Cela a confirmé ma façon de faire de manière collective, même si ces deux années n'ont été, en ce qui me concerne, d'aucun repos ! 
Après ce constat, j'ai fait un vil calcul et j'ai choisi d'enseigner plutôt que de rester au musée, car la recherche universitaire pouvait déboucher sur des mandats de commissaire. Ce fut un passage relativement court, mais bénéfique puisqu'il m'a permis de mettre en œuvre certains éléments théoriques et pratiques appris auparavant. Ce fut une chance exceptionnelle d'analyser, d'étudier et de documenter la collection d'art canadien du Musée des beaux-arts de Montréal dans la perspective de la mettre en valeur et de l'enrichir par le biais d'acquisitions. Ceci a mené à des acquisitions importantes, dont un très beau tableau de Paul Peel (1860-1892), Le Repos ou celui de Joseph Légaré (1795-1855), Québec vu de Pointe de Lévy.

\section{Ce sont finalement vos études en histoire de l'art qui vous ont dirigé vers la muséologie. Quel était le sujet de votre thèse de doctorat?}

J'ai produit une thèse sur un sujet qui n'en était pas un et qui portait sur le fonds de tableaux des abbés Desjardins importés de France à Québec en 1817 et 1820. J'ai amorcé ma thèse en 1973, mais que je ne l'ai déposée qu'en 1998 !

La recherche était énorme parce que la documentation était pratiquement inexistante. Je m'étais donné comme objectif de retrouver les 180 tableaux et d'étudier leur effet sur le développement de la peinture au Bas-Canada en utilisant les notions de transferts culturels et de copie... J'ai trouvé, ce faisant, 250 copies que j'ai aussi dû documenter. Si j'ai pu identifier quelques tableaux importants dans cet ensemble, il s'est révélé être le fait d'artistes français alors peu documentés. Ma thèse a nécessité avant tout un travail de recherche dans les archives, tant sur les tableaux que sur l'histoire du fonds. Parce que je n'y travaillais que l'été, sa production a été assez lente. J'ai aussi dû changer de directeur de thèse puisque le premier, Yves Bottineau, à l'Université de Paris-X Nanterre a quitté son poste. C'est John R. Porter de l'Université Laval qui en a assuré la direction jusqu'au dépôt.

Par un heureux retour des choses, le Musée national des beaux-arts du Québec et le Musée des Beaux-Arts de Rennes se sont associés cet été, pour souligner le bicentenaire du transfert de ces tableaux spoliés lors de la Révolution française, qui ont eu une influence sur notre peinture. J'ai eu l'occasion d'agir à titre de commissaire scientifique pour cette manifestation.

Vous avez été, à titre de commissaire, l'auteur de nombreuses expositions. Quelles ont été pour vous les plus significatives? Quelles sont celles qui semblent être les jalons de votre carrière? Mon parcours s'est fait autour de deux catégories d'expositions portant soit sur l'art historique, soit sur l'art moderne et contemporain. J'ai réalisé ces dernières, plus d'une quinzaine, en collaboration avec des galeries universitaires, des centres d'exposition ou des musées en région, ou en collaboration avec des institutions qui ne produisent habituellement pas d'expositions d'art, comme le Centre Canadien d'Architecture (CCA) ou la Bibliothèque et Archives nationales du Québec 
(BAnQ). L'art contemporain m'intéressait depuis mes études universitaires, mais il ne me semblait pas y avoir beaucoup de débouchés dans ce domaine occupé par la plupart de mes collègues. Je me suis intéressé à Ozias Leduc qui, décédé en 1955, était l'un de nos contemporains. Leduc s'est toutefois révélé être un homme du $19^{\mathrm{e}}$ siècle, un personnage nous ramenant vers le passé parce qu'inscrit dans une démarche artistique plus traditionnelle et ayant des préoccupations esthétiques moins ancrées dans les avant-gardes.

Je me suis donc fait plaisir en montant des expositions consacrées à des artistes ou à des sujets d'art moderne ou contemporain. J'ai ainsi présenté à trois reprises le travail d'Irene F. Whittome (fig. 1) ou celui de Pierre Dorion dans une exposition présentée dans cinq lieux différents. Plus récemment, une mini-rétrospective du travail de Lisette Lemieux était répartie en deux lieux (fig. 2). Fréquenter des artistes d'expérience, comme Micheline Beauchemin ou Guy Pellerin, m'a aussi amené à transposer sur mes objets historiques des réflexions au sujet de la création, de la poïétique, de la solitude du travail d'atelier, des questions qui n'auraient pas existé sans ces expériences. J'ai été aussi amené à réfléchir à la question des réseaux d'artistes, à la dimension sociologique et au contexte de production. Ces collaborations m'ont d'ailleurs amené à m'intéresser aux ateliers qui deviennent des musées. Les expositions produites avec la participation des artistes et de plus petites structures muséales permettent de superviser toutes les facettes de l'exposition depuis sa conception jusqu'aux outils de médiation et de diffusion.

Ce sont des situations différentes des expositions historiques comme celles réalisées sur les arts en Nouvelle-France (2013, MNBAQ), sur la peinture à Montréal entre 1915 et 1930 (1996, MNBAQ, Galerie de l'UQAM) ou sur les œuvres d'Ozias Leduc (1996, MBAM, MNBAQ, MBAO) et de Suzor-Côté (2002, MNBAQ, MBAC) que j'ai préparées en collaboration avec de grandes institutions. Grâce à ces deux personnalités fortes et opposées qui ont opéré à la façon d'une figure de Janus au tournant du $20^{\mathrm{e}}$ siècle, j'ai approfondi mes connaissances de cette période. Ces expositions se sont avérées des projets de recherche de grande ampleur. Elles ont requis la participation de plusieurs acteurs: assistants de recherche, collectionneurs privés, institutions prêteuses, en plus des équipes professionnelles que les musées mettent à la disposition des commissaires. Ces expériences m'ont ainsi fait bénéficier de traditions, d'un savoir-faire et d'une expertise tant sur le plan de la constitution des expositions que de leur diffusion.

\section{Pouvez-vous isoler dans chacune de celles-ci un apprentissage, un concept, une ou des observations que vous avez par la suite incorporés à vos enseignements?}

Puisque mon enseignement en muséologie se concentrait sur la collection et les fonctions muséales liées au collectionnement, l'application de ces expériences s'est faite autrement. C'est en considérant tout le travail d'équipe qu'exige l'exposition dont la recherche et la collaboration du muséographe, des services de gestion des collections, des programmes 


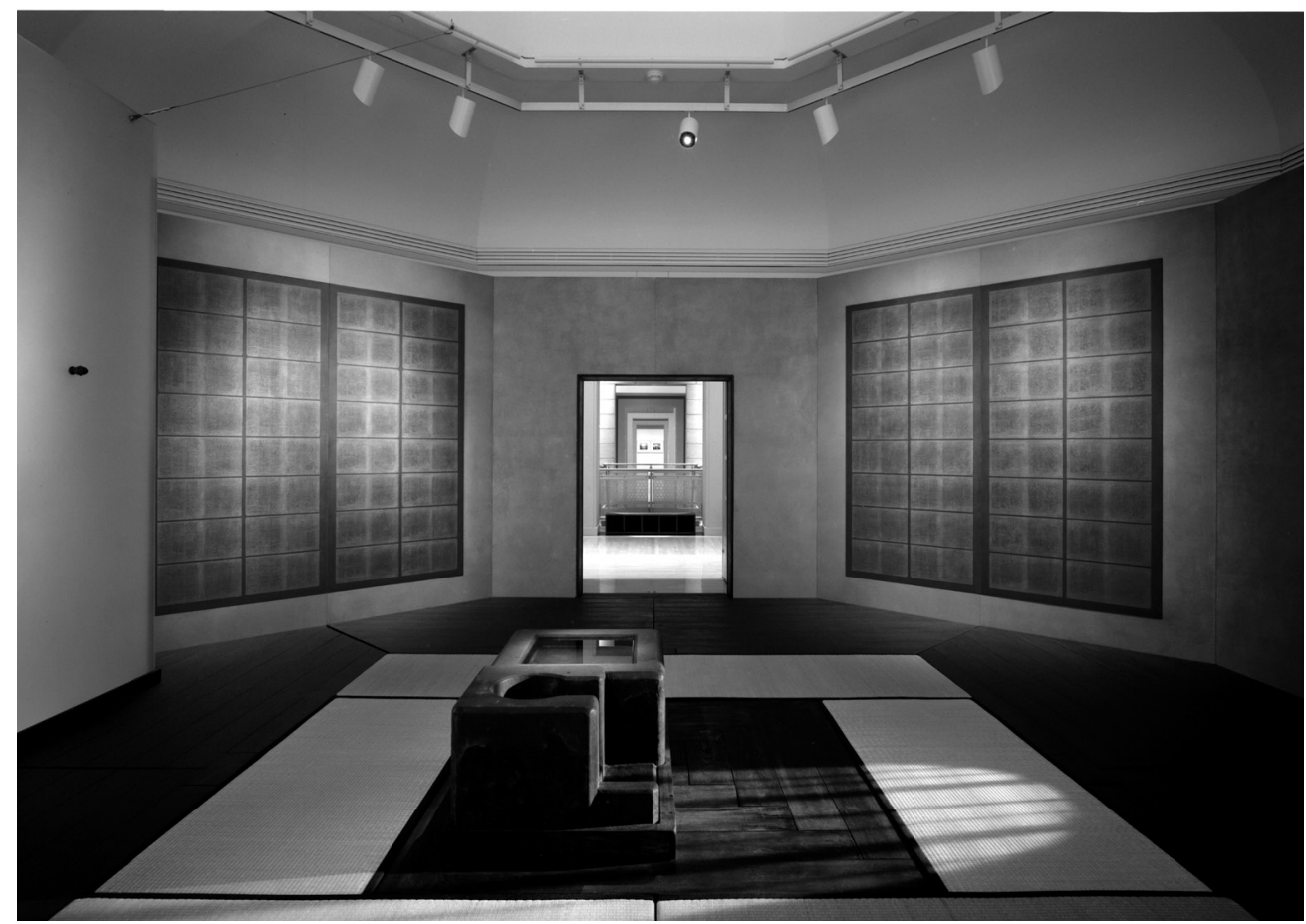

Figure 1

Vue de l'exposition Embarquement pour Katsura: Irene F. Whittome au CCA, 1998

[Photographie: ( CCA]

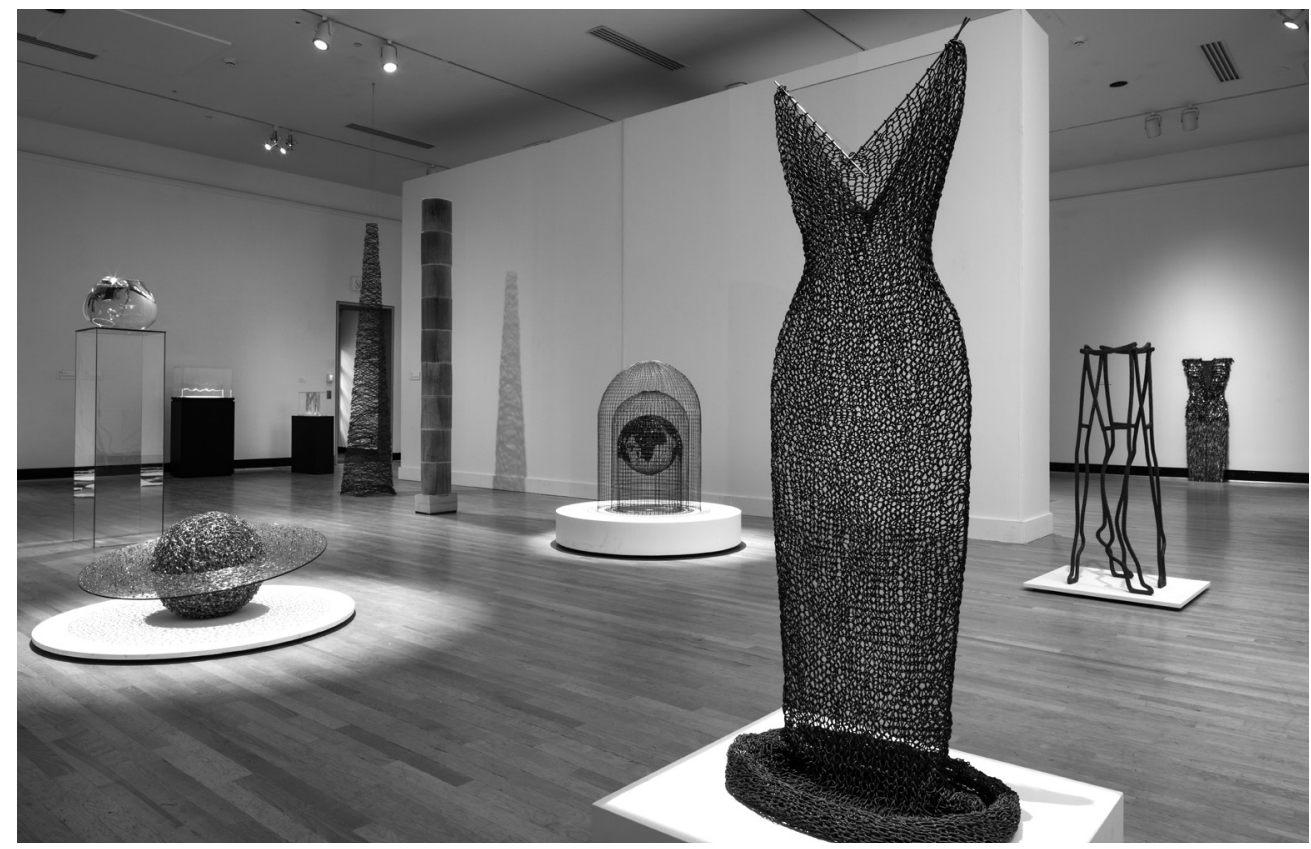

Figure 2

Vue le l'exposition Lisette Lemieux. Empreintes et emprunts, Galerie d'art du Centre culturel de l'Université de Sherbrooke, 2 mars -8 avril 2017.

[Photo: Michel Dubreuil] 
éducatifs et publics, des publications et des relations publiques qui ont, elles aussi, comme objectif de faciliter la mise en valeur de l'exposition et son rayonnement, que l'on peut appliquer cette expertise à la collection.

Dans cette optique, j'ai également intégré la notion de conservation préventive à mon enseignement parce qu'il s'agit d'un volet qui touche directement la gestion et la conservation des collections, mais aussi l'exposition (manutention, transport, conditions d'exposition des différents matériaux, etc). En étroite collaboration avec les restaurateurs du Centre de conservation du Québec (CCQ) et de l'Institut canadien de conservation (ICC), j'ai conçu et produit en 1990, 19 vidéos sur la conservation préventive. Cette série bilingue a été vendue dans une trentaine de pays et est toujours utilisée dans le cadre de la formation en muséologie.

\section{Feriez-vous des amendements à ces vidéos aujourd'hui ?}

Les contenus, ce sont les spécialistes qui les ont fournis et ils ont été validés par l'ICCROM. Nous nous en sommes tenus aux principes de base en gardant à l'esprit les institutions muséales pourvues de peu de ressources. Il s'agissait de structurer cette information en outil pédagogique. J'ai accompagné l'équipe de production et de réalisation lors des tournages et de la postproduction afin d'assurer la cohérence de l'ensemble.

Il y a de nouveaux matériaux et supports qui ne sont pas couverts par la série. Cependant, je ne crois pas que le champ ait évolué au point d'en faire un outil désuet. Il est utile pour la muséologie au sens large et il a son rôle à jouer dans la formation continue. On peut consulter ces vidéos sur le site du CCQ. http://www.ccq.gouv.qc.ca/index.php?id=88

Le programme conjoint de maîtrise en muséologie de l'Université du Québec à Montréal et de l'Université de Montréal fête cette année, ses 30 ans d'existence. Je sais que vous avez grandement contribué à la constitution de ce programme, et que vous y avez joué un rôle central durant plusieurs années. Puis-je vous demander de préciser la nature de votre rôle et votre contribution?

Lorsque je suis arrivé à l'UQAM en 1988, le programme qui avait été pensé et mis sur pied par les professeurs Louise Dusseault-Letocha et Raymond Montpetit existait depuis un an. La direction du programme m'a été confiée en 1989 pour deux mandats de deux ans. Le programme était encore relativement nouveau. Il fallait l'implanter et le faire connaître. Les musées occupaient déjà une place importante dans la sphère culturelle et attendaient l'apport de jeunes professionnels et ils ont été très accueillants.

L'administration de l'UQAM faisait une part importante à la voix étudiante représentée dans les comités d'admission et de programme.

Ce fut l'occasion de formidables collaborations avec les étudiants qui 
se sont énormément impliqués dans la création de ressources (centre de documentation, journées d'étude et mini-colloques). Nous avons pu tenir compte de leurs besoins, tout en bénéficiant de leur énergie.

J'ai travaillé avec eux et les collègues professeurs à consolider le programme, à accroître sa crédibilité afin que les étudiants, lors de leur stage, puissent avoir accès à des institutions de choix, qui correspondent à leurs capacités et aspirations. Nous avons eu la chance d'attirer de jeunes professionnels qui travaillaient dans le milieu et qui venaient parfaire leur formation. Pour les musées hôtes, ces candidats étaient intéressants, ce qui nous a permis de construire les ponts nécessaires afin qu'ils acceptent, au cours des années successives, de recevoir d'autres étudiants et ainsi contribuer à leur développement. Il fallait également réunir le corps professoral. À ses débuts, aucun professeur n'était rattaché au programme de muséologie et nous étions tous " prêtés " par les différents départements. Nous avons pu profiter de l'expertise de professeurs comme Bernard Schiele et Michel Allard et maintenant Yves Bergeron et Jennifer Carter. Avec d'autres chargés de cours provenant du milieu, je pense à Claude Benoît, Arlette Blanchet et Marie-Thérèse Bournival, nous avons contribué au renforcement et à la spécialisation de ce programme.

Cette période s'est également avérée difficile. Mon bilan est mitigé: il m'a fallu composer avec une crise économique qui mettait l'université dans une situation peu enviable tandis qu'en muséologie, nous vivions un boom exceptionnel. C'est à ce moment qu'apparaissent le Musée de la civilisation, le Biodôme, le CCA, que le MNBAQ, le MBAM et le Musée McCord s'agrandissent et que le Musée d'art contemporain de Montréal ouvre ses portes après son déménagement. J'ai cherché à convaincre mes patrons de financer le programme afin de lui donner la possibilité de s'arrimer à tout ce dynamisme, à ce développement que nous voyions passer sous nos yeux... Il a été toutefois impossible de monter dans ce train, sauf de façon bénévole ou en profitant d'occasions favorables. Sans les remettre en cause, ces collaborations n'ont pas eu l'impact d'un travail reconnu et n'ont pas engendré les partenariats dont un financement accru aurait fait profiter le programme. L'incapacité à débloquer des fonds a été l'une de mes grandes déceptions.

\section{Est-ce que les institutions ont toujours répondu à votre appel?}

Il s'agissait alors d'organiser les stages pour les premières cohortes d'étudiants. Les institutions ont bien répondu parce que notre priorité était de les jumeler le plus adéquatement possible aux besoins de formation. Nous pouvions, par exemple, placer des étudiants en stage au bureau de direction du Musée des beaux-arts du Canada, au Musée des beaux-arts de l'Ontario et au MBAM auprès de conservateurs chevronnés, parce que nous avions les candidats qui avaient les aptitudes et le désir de faire un stage à ce haut niveau. 


\section{Le programme a toujours été conjoint, n'est-ce pas?}

Le programme a été conçu conjointement avec l'Université de Montréal: c'était la condition que posait le Ministère pour sa création. Il a été pensé pour permettre de réfléchir sur les principales fonctions du musée et de développer une spécialisation dans une de ses fonctions (collection, exposition, éducation) ou dans un type de musée (d'art, de sciences, de société). Chaque université accueillait, à cette époque, 10 étudiants par année. Le programme était contingenté de façon à ne pas saturer le marché. Les musées de sciences et de société ont adhéré à la formation interdisciplinaire que nous avons mise en place.

J'ai eu la chance d'y retrouver Jean Trudel, directeur à l'Université de Montréal. Nous avons produit la première évaluation du programme, à sa cinquième année d'existence et 25 ans plus tard, la seconde est maintenant produite. Il s'agit d'un processus laborieux et nous ne sommes pas peu fiers d'avoir accompli cette tâche, puisqu'il fallait démontrer la crédibilité du programme et sa pertinence afin de pouvoir le poursuivre et le bonifier.

Votre cheminement a été couronné de nombreux prix, dont le prix Carrière de la Société des musées québécois (1997), le prix Gérard-Morisset (2008) du gouvernement du Québec, la médaille d'honneur de la Faculté des arts et des sciences de l'Université de Montréal (2008). Vous êtes devenu membre du Cercle d'excellence de l'Université du Québec (2009), admis à l'Académie des lettres du Québec (2012), puis nommé professeur émérite de l'UQAM (2015). Vous avez finalement été nommé, l'an dernier, membre du Conseil du patrimoine culturel du Québec et avez reçu l'Ordre du Canada. Cette suite de reconnaissances, liée à votre production intellectuelle et au cumul de vos expériences, vous permet très certainement de formuler un point de vue assez objectif de la situation des musées au Québec et de son évolution depuis votre entrée dans ce milieu (les années 1970 à aujourd'hui). Pouvez-nous nous en faire part?

Ces marques de reconnaissance témoignent, d'une autre manière, de la chance que j'ai eue d'avoir pu faire un métier qui me passionne et dans lequel j'ai pu m'épanouir. Je dirais que le poste d'observation que constituait le Groupe de travail sur l'avenir des musées au Québec et le rapport qui a suivi (2013), rapport cosigné par Claude Corbo, Marie Lavigne et moi-même, a fourni un autre point de vue qui permet également de répondre à votre question.

Au-delà des transformations les plus visibles qui se sont opérées sous l'effet du tourisme de masse et la plus grande accessibilité aux musées, de l'impact des technologies comme alliées dans la transmission des messages, des pressions budgétaires qui forcent les institutions à se tourner vers le financement privé, les musées ont grandement progressé. 
Le premier secteur est sans doute celui des politiques internes. Au cours des premiers séminaires que j'ai donnés, je demandais aux étudiants de collaborer avec une institution dans le but de préparer une politique de gestion et de développement des collections, puis une politique de recherche. Les musées étaient nombreux à ne pas disposer de tels outils. Le but de ces exercices était de réfléchir avec les acteurs de ces institutions, afin de les doter de ces instruments de base qui m'apparaissent essentiels tant au développement d'une vision à long terme qu'en ce qui concerne leur fonctionnement au quotidien. Lorsque ce cadre structurant est déficitaire et que le mandat est trop vaste par rapport aux ressources disponibles, il n'est pas possible de travailler de manière cohérente. J'ai constaté qu'un saut important a été effectué dans ce domaine même s'il reste encore beaucoup de travail à faire pour maintenir ces politiques à jour et les appliquer.

Les mandats ne sont pas toujours définis de façon précise, ce qui permettrait de distinguer les missions des institutions entre elles. Souvent trop larges, ils empêchent la mise en œuvre de politiques spécifiques aux champs investis par les institutions. Même si les mandats sont encore beaucoup trop larges, je crois que les représentants des institutions y réfléchissent de façon plus sérieuse. C'est devenu une préoccupation et la plupart des musées ont établi des politiques. On oublie peut-être de les mettre à jour, mais on s'y réfère de temps à autre.

Il faut souligner, au cours de ces 40 ans, le jalon important que représente la normalisation de la formation professionnelle des institutions muséales. Sur le plan pratique, le rôle joué par le Centre de conservation du Québec et par la formation collégiale en technique muséale a permis que les collections soient entretenues par une main-d'œuvre professionnelle qualifiée. Avoir des techniciens bien formés est l'une des grandes avancées de la muséologie au Québec, qui s'est faite en parallèle avec un travail important sur l'expographie. Le design d'exposition est devenu un nouveau réflexe. Sur le plan conceptuel, la formation universitaire en muséologie a permis d'outiller intellectuellement les professionnels à répondre, non plus uniquement aux cadres imposés par les enjeux propres aux musées, mais bien à une situation culturelle plus étendue.

\section{Est-ce que ce manque de définition des mandats et des politiques muséales est aussi lié au manque de professionnalisation des muséologues?}

Certainement, et l'on constate que les conseils d'administration des musées recrutent des directeurs qui n'ont pas nécessairement une formation en muséologie. La bataille n'est donc pas gagnée; il y a encore du chemin à faire quant à la formation muséale des cadres supérieurs.

L'entrée de l'institution dans un nouvel ordre économique et sociétal, lié à la redynamisation du cadre urbain constitue une évolution qu'on ne pouvait pas prévoir, il y a 40 ans. Dans les années 1970 et 1980, la mise en place des services éducatifs au musée entraînait de vastes débats et de nombreux colloques, forums et grandes rencontres portaient sur cette question qui semble occultée maintenant au profit du marketing, du 
design et de la commercialisation. La question du rôle du musée dans la société a ainsi été déplacée vers les relations publiques, ou vers des problématiques communicationnelles élargies, qui ont supplanté la vocation pédagogique du musée. Maintenant, une exigence communicationnelle axée vers un public élargi est devenue la priorité. Elle est venue détrôner l'éducation et la collection, au point que la collection - l'objet - devient même un poids pour certaines institutions. Cette approche est paradoxale dans la mesure où l'on réalise au même moment que la collection est un atout qui permet de contrecarrer le coût des méga-expositions et une tendance se dessine qui vise à exploiter ce potentiel dans le cadre d'activités événementielles.

La réflexion sur ce que constitue l'institution muséale est le fait d'une relève de mieux en mieux formée. Cependant, nous sommes à la fois victimes et coopérants d'un système où le musée se retrouve investi dans un engrenage socioculturel qui le sépare de son objet premier, soit la conservation et diffusion d'un patrimoine en vue d'une appropriation par les générations futures.

\section{À quels problèmes et défis les musées québécois et canadiens sont-ils aujourd'hui confrontés?}

Je me permets de revenir sur le volet des mandats institutionnels trop larges afin d'expliciter ma pensée. Un constat s'impose. On dénombre au Québec près de 450 institutions qui ont un caractère muséal, centres d'exposition et d'interprétation compris. Même si un certain nombre de musées disparaissent chaque année, dont quelques-uns sont englobés par d'autres institutions, d'autres apparaissent et la croissance semble incontrôlable. Cette effervescence fournit un dynamisme culturel certain, mais constitue en même temps un poids social et économique. Les lieux de diffusion de la culture se sont multipliés et la population de la province demeure somme toute limitée. L'offre est-elle proportionnelle à la demande? Ne serait-il pas possible que ces organismes collaborent en vue de partager mandats, projets, expertise et services? On remarque quelques tentatives de rapprochements, très bénéfiques pour toutes les parties il me semble, mais beaucoup reste à faire. L'exemple dans ce domaine devrait d'ailleurs provenir des musées nationaux.

Je constate, également qu'au nom de l'interdisciplinarité, fort positive en soi, que les musées de société collectionnent et exposent des œuvres d'art et que les musées d'art adoptent des thématiques qui viennent empiéter sur le champ de compétences des musées de société. Cette porosité des champs fait en sorte que les mandats et la mission des institutions tendent à s'élargir. Cela pose problème dans le sens où les musées qui adoptent ce mode opératoire travaillent de façon ponctuelle avec des spécialistes pour enrichir leur propos interdisciplinaire, sans construire d'équipes qui permettraient d'approfondir les champs investis. Une fusion d'institutions permettrait de penser plus adéquatement ces approches pluralistes que demande le public. De plus, la collaboration muséale avec les lieux de savoir, dont les universités et les cégeps, n'est pas aussi affinée qu'elle devrait l'être. Il y a bien 
évidemment des initiatives, mais il y aurait avantage à normaliser cette pratique, à établir des protocoles et à construire un réseau, afin d'améliorer l'efficience des mécanismes en place.

Une autre problématique qui m'intéresse, c'est celle d'une collection "nationale ", si tant est qu'il y ait une nation. Quoi qu'il en soit, je crois qu'il serait approprié de mettre en place un réseau muséal québécois et pourquoi pas canadien, en collaboration avec certaines institutions et pluridisciplinaire qui mettrait en valeur et partagerait les collections. Cette coopération, qui inclurait notamment une réflexion d'ensemble sur les mandats de collectionnement - et d'acquisition -, rendrait l'offre muséale plus cohérente, notamment en intégrant l'offre muséale dans toutes les régions. Elle pourrait aller jusqu'au transfert ou jusqu'à l'échange de certains objets, savoirs et compétences entre les institutions. Cette réflexion collective pourrait, par exemple, porter sur les expositions en proposant des thématiques communes. Chaque musée pourrait, le cas échéant, exploiter un sous-thème spécifique correspondant à son propre mandat institutionnel et adapter la proposition commune à l'expérience que l'on souhaite faire vivre aux visiteurs auxquels on s'adresse. Dans le contexte des parcours touristiques, cette structure offrirait une pertinence renouvelée à la visite des institutions sur l'ensemble du territoire et pourrait nourrir la muséologie scientifique qui est en réel déficit au Québec.

Cette collaboration entre musées pourrait impulser la création d'expositions collectives itinérantes qui seraient plus riches. Parce que les expositions sont souvent des projets déjà répartis dans des plans quinquennaux, il serait possible de les construire en cohésion entre les institutions et d'offrir une logique conceptuelle qui dépasse les murs d'un seul musée pour rejoindre une grande partie du réseau. Il faut d'ailleurs rappeler que les institutions sont payées par les contribuables, que ce soit par l'aide directe des différents paliers de gouvernement ou par l'appui du secteur privé qui y trouve des avantages fiscaux, et qu'elles doivent s'affairer à être accessibles aux publics qu'elles desservent. En réduisant au minimum l'expertise muséale au sein du ministère de la Culture et des Communications, le gouvernement provincial s'est délesté de son leadership dans ce domaine au lieu de participer au développement d'une vision d'ensemble qui fédère les actions des nombreux ministères (Affaires municipales, Tourisme, Sciences et Innovation, Éducation et enseignement supérieur, Relations internationales... ) qui ont à voir avec le musée.

Outre le patrimoine scientifique, le patrimoine autochtone et le patrimoine religieux demeurent des problèmes entiers puisqu'une solution d'ensemble n'a toujours pas été trouvée pour ces secteurs. La résurgence des populations d'origine amérindienne qui exigent de prendre part à la définition de la culture s'établit au même moment où l'on remarque la disparition des communautés religieuses et la fermeture de paroisses. Il n'y a toujours pas de réponse globale qui permettrait une répartition et une diffusion cohérente de ces patrimoines où la dimension régionale 
est essentielle. Dans ces secteurs orphelins, comme ceux qui sont déjà couverts, l'adaptation des messages et l'attention à porter aux publics sont primordiales.

\section{Pouvez-vous identifier les enjeux auxquels font face les grands musées par rapport à ceux dont la taille est réduite?}

Ces enjeux sont liés à la reconnaissance publique et à leur capacité de constituer des réseaux. Les grands musées ont plus de visibilité et peuvent donc s'inscrire dans un système plus large de collaboration et de ressources externes. Les plus petits musées, qui disposent de moins de moyens et de conseils d'administration moins avertis et influents, ont moins d'impact. Ce sont les membres du CA qui peuvent aider à construire des partenariats intéressants. La direction peut en faire une partie, mais les multiplicateurs sont vraiment les membres du conseil. Un des problèmes au Québec, c'est qu'il y a trop de petits musées qui exigent la présence de ces nombreux bénévoles d'expérience et pas assez de musées de taille moyenne.

\section{Quel avenir entrevoyez-vous pour les musées en région?}

Je fais la différence entre une région administrative et une région culturelle. Cette dernière se base sur une histoire, une géographie, une culture, qui vient d'un passé commun à un groupe, à une communauté. C'est à celle-ci que je me reporte et à partir de laquelle il faut travailler, alors que le financement est réparti par région administrative. Je tiens à souligner cette réalité parce qu'on voit parfois émerger des musées ayant des mandats similaires, mais qui sont subventionnés par deux régions administratives géographiquement voisines.

Le Québec est composé des identités portées par ses différentes régions culturelles. Nous avons besoin de centres régionaux forts, de ces lieux qui communiquent ce que nous sommes dans cette partie du monde. Ce rôle identitaire est souvent ce qui constitue l'essence des ces musées. Nous ne pouvons donc pas concevoir une muséologie saine sans d'importants musées régionaux.

Je crois en leur avenir parce qu'ils semblent avoir compris qu'ils devaient faire front avec leurs municipalités respectives, et que celles-ci ont compris à leur tour qu'elles devaient collaborer avec leur musée pour la mise en valeur de leur environnement. On y pratique souvent une muséologie dynamique sur le plan de la médiation, par des expériences muséales qui n'ont certes pas de grands moyens, mais qui sont souvent originales. Leurs collections sont riches, souvent peu documentées, mais il y a là un potentiel énorme à connaître et à mieux faire partager.

Puisque vous avez acquis une certaine expérience des conseils d'administration des institutions muséales, pouvez-vous nous décrire votre rôle au sein de ceux-ci, et exprimer votre avis sur l'état actuel de ces conseils au Québec?

Lorsque j'étais vice-président de la Société des musées québécois (SMQ de 1990 à 1993), j'ai travaillé à une meilleure formation des membres des conseils d'administration. Je suis convaincu qu'ils sont la clé de 
voûte du système muséal, car ce sont eux qui choisissent les directeurs des institutions. Il est certain que le gouvernement devrait être plus exigeant quant aux aptitudes et à la formation des membres des conseils d'administration des institutions qu'il subventionne. La SMQ et l'Association des musées canadiens ont conçu des outils sur les compétences à posséder et la composition d'un conseil d'administration, mais il s'agit de ressources que les présidents de ces conseils ne consultent, voire ne connaissent pas nécessairement.

C'est au cours de leurs réunions que se prennent les décisions importantes sur l'orientation et les perspectives d'avenir de l'institution. Si les membres sont compétents, intéressés, formés, dévoués, le musée et le système qui le soutient se développent de façon formidable. Les postes des conseils d'administration sont bénévoles et souvent honorifiques et ceux qui sont nommés représentent déjà une entreprise, un organisme ou ont accompli quelque chose de remarquable. Ceux qui dirigent ces conseils doivent comprendre la valeur et la pertinence de chacun de leurs membres, et si ceux-ci ne répondent pas à leurs obligations, ils doivent être remplacés. Au-delà de l'incontournable appui à la direction pour divers projets, ces conseils sont mis en place afin d'exploiter le réseau de chacun des membres pour contribuer à des dossiers spécifiques.

Pour ma part, j’ai joué différents rôles selon que le musée existait déjà, venait d'être créé ou était en processus de création. J'ai souvent rempli le rôle de muséologue de service, parce le conseil était constitué de gens d'affaires et de membres dont la compétence n'était pas la muséologie. J'ai été invité à siéger notamment aux conseils d'administration du Musée d'art de Saint-Laurent, de l'Écomusée du fier-monde, du Château Dufresne, du Musée des arts du spectacle vivant et maintenant de la Fondation Guido Molinari. Mon rôle a souvent été celui d'agent de liaison entre la direction et les membres du conseil sur les enjeux muséologiques, dont le développement des collections et la politique d'expositions.

Après plusieurs années d'interruption, le Québec bénéficie de nouveaux espaces muséaux. Le Musée des beaux-arts de Montréal a ouvert deux nouveaux pavillons et une salle de concert (le pavillon Claire et Marc Bourgie de l'art québécois et canadien, et la salle de concert Bourgie, le pavillon pour la paix Michal et Renata Hornstein) en moins de cinq années d'écart. Le Musée national des beaux-arts du Québec a ouvert le pavillon Pierre-Lassonde dédié à l'art contemporain et aux expositions temporaires. S'ils permettent une exposition plus éloquente des collections de ces institutions, et favorisent l'implantation de nombreux programmes d'enrichissement culturel et public, ces pavillons sont aussi des apports significatifs dans les stratégies de revitalisation de chacune de ces villes. Pouvez-vous nous donner votre avis sur ces nouvelles structures?

Vos exemples se rapportent à deux grandes villes. De nombreuses autres constructions ou agrandissements de musées ont eu cours récemment, comme en fait foi l'ouvrage Architectures d'exposition au Québec (2016). 
Je ne vais pas formuler de jugement sur les bâtiments en tant que tels, mais je me permets d'interroger l'évaluation des besoins du Québec et l'analyse des collections et des services avant de procéder à la construction de ces structures. Je pense qu'il s'est fait des agrandissements narcissiques, c'est-à-dire que certains musées se sont agrandis pour satisfaire leur propre image sans tenir compte de leur place dans l'écosystème culturel dans lequel ils s'inscrivent, de leurs capacités à faire plus avec ce qu'ils possédaient déjà, et sans penser à combler des vides ailleurs dans le réseau québécois ou à mesurer leur impact global.

Est-ce que l'enrichissement passe nécessairement par la construction de nouveaux espaces? La question mérite d'être posée. Ne devrait-on pas plutôt, au Québec, dynamiser nos collections en développant des expositions itinérantes à partir des meilleures ressources en vue de répondre aux questions sociétales qui se posent? Je crois que l'effet Bilbao, qui semble dans certains cas le résultat recherché, s'essoufflera à plus ou moins long terme et que nous nous retrouverons avec davantage d'espaces à entretenir et à financer. Il est vrai qu'une partie importante de ces architectures consiste en locaux à louer ou en services paramuséaux (restaurants, boutiques) qui garantissent une source de revenus pour les musées.

\section{Quelles sont, selon vous, les grandes transformations qui ont marqué la muséologie en tant que discipline universitaire et en tant que pratique au Québec?}

La plus importante transformation, celle que permettent les études avancées en muséologie, est le fait de penser le musée au Québec comme objet d'étude, pour le définir et, éventuellement, le redéfinir souvent de manière comparative. Certains travaux dirigés, les mémoires et thèses en muséologie, en communication, en histoire, en éducation, par exemple - car la muséologie est un secteur d'études dans plusieurs départements -, favorisent une meilleure compréhension des fonctions du musée, de ses outils et de son rôle. Ces études accompagnent le développement des musées. Elles participent à ce développement grâce à cette réflexion conceptuelle.

La recherche est souvent appliquée et les études de cas servent à repenser le musée en lui permettant de s'adapter aux métamorphoses qu'il connaît. La muséologie permet de réfléchir l'objet-musée relativement à son territoire, mais aussi à ses idéologies et aux valeurs qu'il porte. Les résultats sont souvent de nature expérimentale, mais ils s'inscrivent dans un mouvement d'ensemble qui montre bien la place centrale qu'occupe maintenant le musée dans le tissu culturel et social.

Enfin, comment entrevoyez-vous l'avenir des musées? Quelles sont, selon vous, les mutations structurelles et culturelles qui s'opéreront sur ces entités, et dans quelles perspectives?

La chance qui m'a accompagné jusqu'à maintenant ne m'a pas doté des qualités de devin. Les enjeux sont nombreux et les tentations de s'engager dans des voies stériles sont omniprésentes. Cependant, il faut demeurer positif en dépit de certaines tendances qui pointent vers une 
programmation d'expositions souvent moins exigeante qu'on le souhaiterait, un collectionnement tous azimuts, et un travail en silo et compétitif entre les musées qui doivent lutter pour leur survie en oubliant trop souvent d'exploiter les voies de la collaboration qui seraient pourtant profitables pour eux et le public.

Le Groupe de travail sur l'avenir du réseau muséal québécois proposait des pistes de solution à court et moyen termes, adressées au gouvernement et aux musées pour orienter leur compréhension de la situation, tant sur le plan de la culture gouvernementale et du financement, que sur le plan de la collaboration régionale - la Capitale nationale et Montréal sont aussi des régions - et l'implication des partenaires sur l'ensemble du territoire. Les parties ont préféré ignorer les recommandations de ce rapport, maintenir le statu quo et procéder à des aménagements mineurs.

Je ne rappellerai pas les problèmes déjà bien ciblés qui portent sur la diversité culturelle de nos sociétés, sur l'importance de l'éducation comme lieu d'initiation à la culture, sur le rôle des technologies de pointe et sur l'importance du rôle et de l'appui de l'État dans la vie de l'esprit, des sujets déterminants pour l'avenir des musées.

Il me semble important d'insister sur le fait que les musées doivent prendre le temps de mieux faire comprendre leur raison d'être, leur mandat et leur rôle dans la société. En d'autres termes qu'ils ne cherchent pas à se confondre avec d'autres institutions sociales et culturelles. L'image d'un centre de loisirs pratiquant une forme d'edutainment s'impose de plus en plus au détriment de sa particularité comme lieu de mémoire, porteur de savoirs et de valeurs liés aux pièces collectionnées. Les messages de ces spécimens et de ces objets se prêtent à de nombreuses interprétations et leur contenu demande d'être toujours reformulé pour demeurer pertinent et accessible. C'est à cette tâche d'interprétation et de traduction que le musée doit s'employer sans cesse. Ce dernier a le devoir de stimuler la curiosité, de susciter l'étonnement et de favoriser les échanges au contact des objets qui lui donne son caractère unique. C'est un défi permanent. 


\section{Bibliographie}

Laboratoire de l'intime. Les natures mortes d'Ozias Leduc, Joliette: Musée d'art de Joliette, 2017, 40 p. Également publié en anglais sous le titre Confidential Experiements. The Still Lifes of Ozias Leduc.

Les arts de Nouvelle-France, Québec: Musée national des beaux-arts du Québec, Éditeur officiel du Québec, 2012, 296 p.

"Le musée de l'Institut canadien de Montréal (1852-1882): un projet inachevé ", Les Cahiers des Dix, $\mathrm{n}^{\circ} 64,2010$, p. 245-290.

Micheline Beauchemin, Montréal: Les éditions du passage, 2009, $192 \mathrm{p}$.

"La collection comme temps de la Nation. Les premières acquisitions du Musée de la province de Québec en 1920 ", Les Cahiers des Dix, no 62, 2008, p. 123-151.

«L'atelier-musée, paradoxe de l'expérience totale de l'œuvre d'art ", Anthropologie et Sociétés, numéro thématique "La culture sensible ", vol. 30, n 3, 2006 [2007], p. 28-44.

L'Espace-couleur de Robert Wolfe, Montréal:

Bibliothèque et Archives nationales du Québec, Québec: Presses de l'Université Laval, 2006, 142 p.

Suzor-Coté Lumière et matière, Montréal: Éditions de l'Homme, Musée du Québec et le Musée des beaux-arts du Canada, Montréal, 2002, 383 p. (aussi publié en anglais sous le titre Suzor Coté Light and Matter).

Pierre Dorion, Sherbrooke: Galerie du Centre culturel de l'Université de Sherbrooke, 2002, $94 \mathrm{p}$.

Embarquement pour Katsura, Irene F. Whittome au C.C.A., Montréal: Centre canadien d'architecture, 1998, $36 \mathrm{p}$.
Ozias Leduc Une œuvre d'amour et de rêve, (LACROIX Laurier dir.), Montréal: Musée des beaux-arts de Montréal, 1996, 318 p. (également publié en anglais sous le titre Ozias Leduc A Work of Love and Reverie).

La conservation préventive dans les musées, (LACROIX Laurier dir.), Guide d'accompagnement d'une série de 19 vidéos, Montréal: UQAM, Service de l'audiovisuel, 1996.

Peindre à Montréal 1915-1930 Les Peintres de la Montée Saint-Michel et leurs contemporains, (LACROIX Laurier dir.), Québec: Musée du Québec-Galerie de l'UQAM, 1996, 120 p. (également publié en anglais sous le titre Painting in Montreal 1915-1930).

«Musées et collections: Impact des collections massives ", Musées et collections: Impact des collections massives, série Conférences + Colloques, Montréal: Musée d'art contemporain de Montréal, 1995, p. 49-58.

"Les musées du Québec. Vingt ans d'essor! Vingt ans de misère? ", Muse, vol. X, nos 2-3, été-automne 1992 (spécial ICOM), p. 111-115 (trad. angl. p. 116-119).

"Les musées de demain, dès aujourd'hui", Musées, vol. 14, n 3, septembre 1992 (spécial ICOM), p. 92-94 (trad. angl. p. 95-96).

"La recherche et les collections, un antagonisme?", dans La recherche et les collections: où en sommes-nous?, Société des Musées québécois, 1990, p. 9-15.

Irene F. Whittome, Parcours dessiné, Hommage à Jack Shadbolt, Musée d'art de Joliette: Éditions Parachute, 1989, 122 p. (édition bilingue)

François Baillairgé (1759-1831) Un portefeuille de dessins académiques, (LACROIX Laurier dir.), (aussi édition anglaise), Galeries d'art Concordia, 1985, $63 \mathrm{p}$.

Dessins inédits d'Ozias Leduc/Ozias Leduc The Draughstman, (LACROIX Laurier dir.), Montréal: Sir George Williams Galleries of Concordia University, 1978, 168 p. 